\title{
Role of Diffusion Tensor Imaging in Functional Assessment of Transplant Kidneys at 3-Tesla MRI
}

\author{
Thambidurai $S^{1} \quad$ Venkatesh Kasi Arunachalam¹ \\ ${ }^{1}$ Department of Radiology, Kovai Medical Center and Hospital, \\ Coimbatore, Tamil Nadu, India
}

J Gastrointestinal Abdominal Radiol ISGAR:2020;3(suppl S1):S7-S14

\begin{abstract}
Address for correspondence Venkatesh Kasi Arunachalam, MBBS, DMRD, DNB, FRCR, Department of Radiology, Kovai Medical Center and Hospital, Coimbatore-14, Tamil Nadu, India (e-mail: drkasivenkatesh@yahoo.co.in).
\end{abstract}

\begin{abstract}
Keywords

- diffusion tensor imaging

- functional assessment

- transplant kidneys
\end{abstract}

Objectives The main purpose of this article is to measure the fractional anisotropy (FA) and apparent diffusion coefficient (ADC) values of cortex and medulla of renal allograft using 3-Tesla diffusion tensor imaging (DTI) in renal transplant patients with normal and graft dysfunction and to assess the correlation between diffusion tensor parameters (ADC and FA) and the estimated glomerular filtration rate (eGFR) value.

Materials and Methods Fifty renal transplant recipients who received either living or cadaveric renal allografts were included in the study. Blood samples for serum creatinine and eGFR value were taken on the same day prior to the magnetic resonance study and the patients were assigned to three groups (A, B, C) according to allograft function (eGFR levels). The mean ADC and FA values of the cortex/medulla in upper, mid, and lower poles were calculated from the DTI sequence. Statistical analysis was performed using paired sample Student's t-test and one-way analysis of variance test. Results The mean ADC values of the cortex were higher than the medulla that was statistically significant. However, the mean FA values were significantly higher in the medulla than the cortex. Mean ADCs and FA of the renal cortex and medulla were significantly higher in group $A$ patients with normal renal function than in group $B$ and $C$ with poor renal functions. The corticomedullary difference in the FA values was more in group A. However, this difference was lower in group B and more so in group C.

Conclusion $A D C$ and FA values in the renal cortex and medulla exhibit a good correlation with allograft function and were significantly lower in transplants with dysfunction than those with good function. FA values appear to be more sensitive than eGFR and ADC for detection of early pathological changes in the graft dysfunction.

\section{Introduction}

Over the last few decades, chronic kidney disease (CKD) has been recognized as a major global public health problem. The approximate incidence of end-stage renal disease is 150 to 200 per million population. Kidney transplantation is the best form of kidney replacement therapy available for patients with end-stage renal failure. ${ }^{1}$ In India, nearly 3,500 transplants are done annually. ${ }^{2}$

DOI https://doi.org/ 10.1055/s-0040-1709084 ISSN 2581-9933.
Despite improvement in surgical techniques and medications used to prevent transplant rejection, some damage is seen in $\sim 95 \%$ of cases 1 year after transplantation. ${ }^{3}$ Current blood tests are insensitive to early kidney damage, so preplanned kidney biopsy following transplantation (protocol biopsy) is recommended to detect damage, despite recognized complications (bleeding, arteriovenous fistula).

Because of the limitations of serum markers and biopsy, imaging can play an important role in the identification of early

(c) 2020. Indian Society of Gastrointestinal and Abdominal Radiology. This is an open access article published by Thieme under the terms of the Creative Commons Attribution-NonDerivative-NonCommercial-License, permitting copying and reproduction so long as the original work is given appropriate credit. Contents may not be used for commercial purposes, or adapted, remixed, transformed or built upon. (https://creativecommons.org/licenses/by-nc-nd/4.0/)

Thieme Medical and Scientific Publishers Pvt. Ltd., A-12, 2nd Floor, Sector 2, Noida-201301 UP, India 
renal damage. Ultrasound and computed tomography provide good anatomic images but limited functional information. Nuclear medicine examinations provide functional information but lack spatial resolution. Magnetic resonance imaging (MRI) has the unique ability to show both structure and function. ${ }^{4,5}$

Renal MRI is shifting from pure visualization of anatomy to assessment of physiologic and functional parameters of the kidney. Functional renal imaging techniques, such as contrast-enhanced MR renography and unenhanced techniques, such as diffusion-weighted imaging (DWI), diffusion tensor imaging (DTI) and blood oxygen level-dependent (BOLD) imaging have shown considerable promise in the evaluation of renal function. ${ }^{6}$

The role of DWI in the evaluation of renal function has recently become a subject of exploration. Apparent diffusion coefficient (ADC) is a quantitative parameter calculated from DWI that combines the effects of capillary perfusion and water diffusion. Anisotropic properties of tissues can be best evaluated using DTI, which, unlike DWI, allows the analysis of diffusion along multiple directions. DTI can quantify how water molecules within the kidney are affected by the body's attempt to reject the transplanted kidney.

In our study, we have evaluated the DTI parameters (ADC, fractional anisotropy [FA]) of transplanted kidney using 3-Tesla and assessed the graft dysfunction by correlating these parameters with patient's estimated glomerular filtration rate (eGFR) value.

\section{Materials and Methods}

This study was a prospective study and has been approved by the institutional ethics and scientific committee; written informed consent was obtained from all patients. Patients who received either living or cadaveric renal transplants attending the nephrology outpatient department and on regular follow-up were included in this study.

\section{Study Design}

Blood samples (for serum creatinine and eGFR value) and MR study were taken on the same day. Patients were assigned to three groups according to allograft function (eGFR levels): Group A was composed of patients with good allograft function (normal to mildly decreased, according to CKD stage 1 to 2 [eGFR $>60 \mathrm{~mL} / \mathrm{min} / 1.73 \mathrm{~m}^{2}$ ]), whereas patients with moderately impaired renal function (CKD stage $3 \mathrm{~A}$ and $3 \mathrm{~B}$ [eGFR $30-60 \mathrm{~mL} / \mathrm{min} / 1.73 \mathrm{~m}^{2}$ ]) were assigned to group B. Patients with severely decreased function (CKD stage 4 and 5 [eGFR $<30 \mathrm{~mL} / \mathrm{min} / 1.73 \mathrm{~m}^{2}$ ]) were assigned to group $\mathrm{C}$.

eGFR was calculated using Modification of Diet in Renal Disease equation. The formula is based on patient's age, race, gender, and serum creatinine level. The normal eGFR is $\sim 100 \mathrm{~mL} / \mathrm{min} / 1.73 \mathrm{~m}^{2}$. eGFR of a well-functioning transplanted kidney is only between 60 and $90 \mathrm{~mL} / \mathrm{min} / 1.73 \mathrm{~m}^{2}$ since it is a single functioning kidney. ${ }^{4}$

Fifty renal transplant recipients (mean age, 47.1 years), composed of 34 men (mean age, 46.5 years) and 16 women (mean age, 48.4 years), who received either living or cadaveric renal allografts, were included in this study. The time interval between renal transplantation and the MR examination ranged between 2 weeks and 10 years, with a median of 30 months.

\section{Inclusion and Exclusion Criteria}

As contrast material was not given, patients were included irrespective of their allograft functional status. Patients with contraindications for MRI scan (pacemakers, severe claustrophobia, MRI incompatible implants, and foreign bodies), immediate postoperative period, and uncooperative patients were excluded from the study. Children ( $<12$ years age) and elderly patients ( $>80$ years) were excluded from the study. After acquisition of MRI, DTI images were evaluated for artifacts and if the images were not satisfactory, they were also excluded from this study.

All patients were examined in the supine position with a 3-Tesla MR scanner (Skyra; Siemens, Germany) with an 18 channel 6-element body coil and spine coil integrated into the imager table. The mean duration of the procedure was 12 minutes and the following sequences were used:

1. Transverse T2-half-Fourier acquisition single-shot turbo spin echo sequence (repetition time msec/echo time msec 1,800/96; section thickness $4 \mathrm{~mm}$; field of view $400 \times$ $320 \mathrm{~mm}^{2}$ ).

2. Echo-planar DTI sequence was performed in coronal plane by using the following imaging parameters: repetition time msec/echo time msec 1,500/90; section thickness $3 \mathrm{~mm}$; field of view, $400 \times 340 \mathrm{~mm}^{2} ; \mathrm{b}=0$, 400 , and 800 second $/ \mathrm{mm}^{2} ; 20$ diffusion directions; echo spacing, 0.61 millisecondsec; band-width $2058 \mathrm{~Hz} /$ pixel. Acquisition time is 6 minutes 23 seconds. No respiratory gating was used because motion is negligible in transplanted kidney owing to its location in the iliac fossa. ADC and FA maps were generated for each patient (-Fig. 1).

\section{DTI Analysis and Data Collection}

Six ellipsoid regions of interest (ROIs) of $\sim 10$ to 15 pixels were placed in the cortex and medulla of the upper pole, mid-zone, and lower pole of each kidney in each patient on parametric ADC map and FA maps (-Fig. 2). The mid-coronal image was used during ROI placement. The mean FA and ADC values were determined separately for the cortex and medulla. All these patients underwent renal biopsy and histopathology examination was performed.

\section{Statistical Methods}

Statistical analysis was performed using paired sample Student's $t$-test to assess the difference between the cortical and medullary DTI values. $p$-Values of less than 0.05 were regarded as statistically significant. To correlate the eGFR values with ADC and FA of cortex and medulla of the three groups and to assess the significance, one-way analysis of variance with post hoc analysis and Bonferroni correction was used.

\section{Results}

There were totally 51 patients. One patient was excluded from the study due to intense motion artifacts. The remaining 

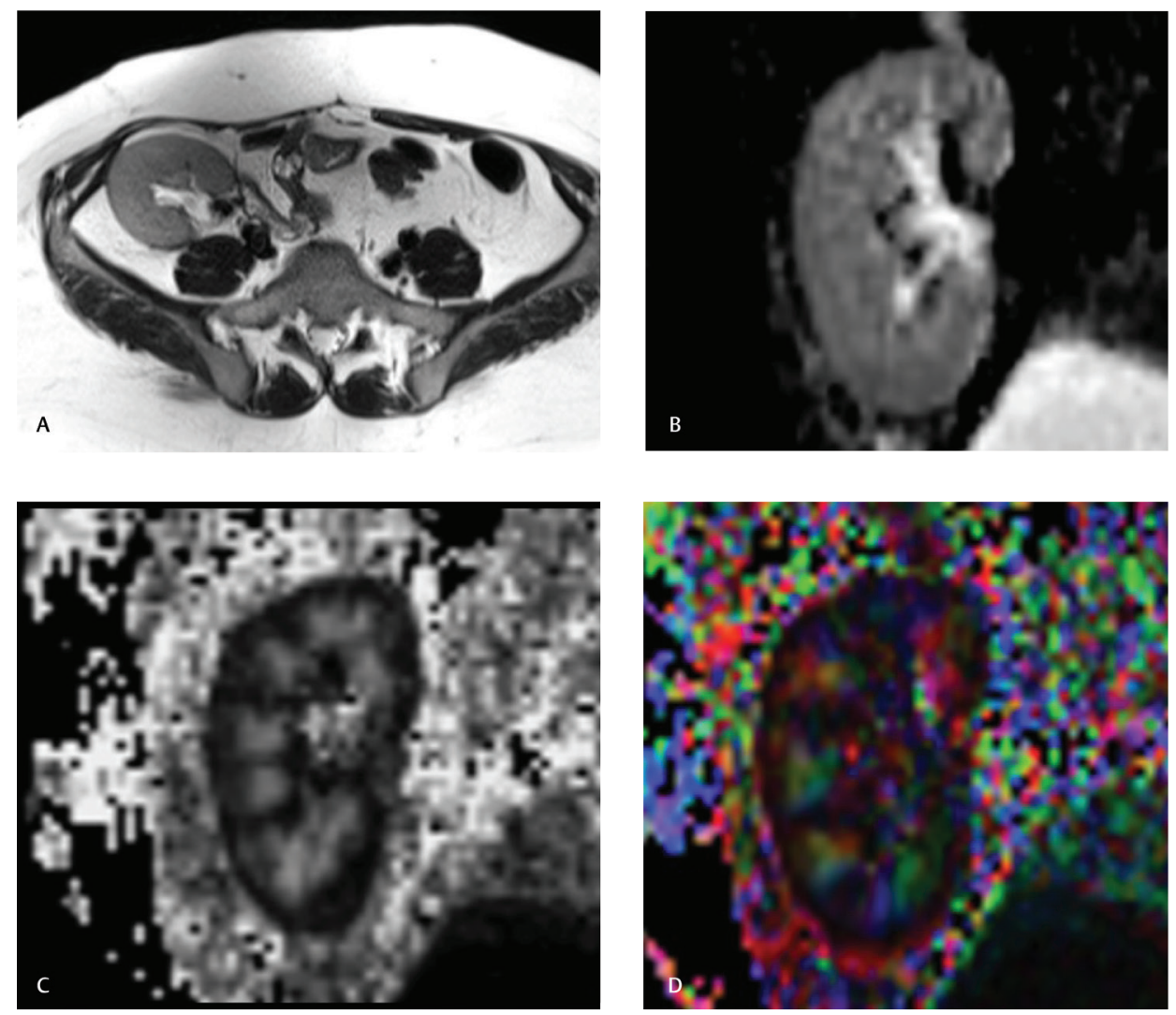

Fig. 1 Representative images in a renal allograft recipient. (A) T2 half-Fourier acquisition single-shot turbo spin echo axial image shows transplant kidney in the right iliac fossa. (B) Apparent diffusion coefficient map of the allograft. (C) Diffusion tensor imaging (DTI) fractional anisotropy (FA) map of the graft. (D) DTI FA color map.
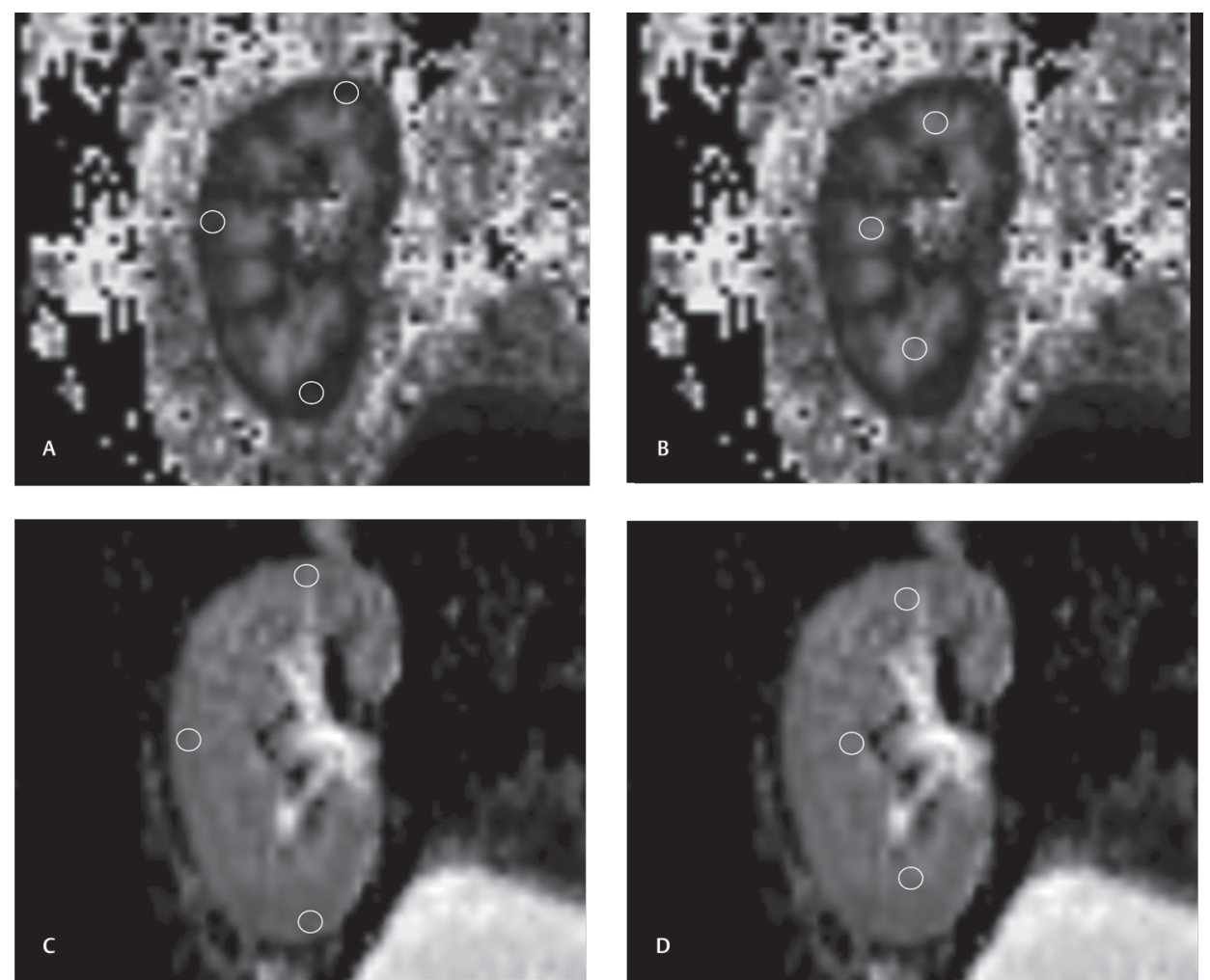

Fig. 2 Examples of region of interest (ROI) placement in the transplant kidney. (A) Diffusion tensor imaging fractional anisotropy (FA) map shows elliptical ROIs placed in the cortex of upper, mid, and lower poles (B) Similar ROls are placed in the medulla of FA map (C) and (D) apparent diffusion coefficient maps showing ROIs in the cortex (C) and medulla (D) of upper, mid, and lower poles. 
50 patients were included in the DTI analysis. The sample of 50 patients was divided into three groups according to the eGFR values (group A-eGFR > $60 \mathrm{~mL} / \mathrm{min} / 1.73 \mathrm{~m}^{2}$ ) and those with poor function (group B-eGFR 30-60 and group C-eGFR $<30 \mathrm{~mL} / \mathrm{min} / 1.73 \mathrm{~m}^{2}$ ).

Group A included 23 patients (mean age, 44.2 years), composed of 19 men (mean age, 44.8 years) and 4 women (mean age, 41.2 years 69.5); group B included 15 patients (mean age, 49.3 years), composed of 8 men (mean age, 44 years) and 7 women (mean age, 55.4 years); group $\mathrm{C}$ included 12 patients (mean age, 49.9 years), composed of 7 men (mean age, 54 years) and 5 women (mean age, 44.2 years).

The mean ADC of the cortex $\left(1783.6 \times 10^{-6} \mathrm{~mm}^{2} / \mathrm{s} \pm 89.2\right)$ $(p<0.001)$ was higher than the medulla $\left(1730 \times 10^{-6} \mathrm{~mm}^{2} / \mathrm{s} \pm\right.$ $88.1)$ that was statistically significant $(p<0.001)$. On the other hand, the meanFA was significantly higher in the medulla( $0.31 \pm$ $0.07)$ than in the cortex $(0.15 \pm 0.02)(p<0.001)$ (-Table 1).

Mean ADCs of the renal cortex were significantly higher in group $A\left(1,868.3 \times 10^{-6} \mathrm{~mm}^{2} / \mathrm{s} \pm 19.3\right)$ than in group $B$ $\left(1752.7 \times 10^{-6} \mathrm{~mm}^{2} / \mathrm{s} \pm 19.2\right)(p<0.001)$ and group $C(1660 \times$ $\left.10^{-6} \mathrm{~mm}^{2} / \mathrm{s} \pm 36.2\right)(p<0.001)$. Similarly in the medulla also the mean ADC was significantly higher in group A (1814.3 $\times$ $\left.10^{-6} \mathrm{~mm}^{2} / \mathrm{s} \pm 24.7\right)$ than in group $B\left(1694.4 \times 10^{-6} \mathrm{~mm}^{2} / \mathrm{s} \pm\right.$ $21.9)(p<0.001)$ and group $C\left(1612 \times 10^{-6} \mathrm{~mm}^{2} / \mathrm{s} \pm 34.7\right)$ $(p<0.001)$ (-Fig. 3).

Mean FA of the renal cortex was significantly $(p<.001)$ higher in group $\mathrm{A}(0.18 \pm 0.01)$ than in group $\mathrm{B}(0.14 \pm 0.01)$ and group $C(0.12 \pm 0.01)$. In the medulla, also group $A$ showed significantly $(p<0.001)$ higher mean FA $(0.39 \pm 0.01)$ than in group $\mathrm{B}(0.26 \pm 0.01)$ and group $\mathrm{C}(0.23 \pm 0.02)$ (-Fig. 4$)$.

The corticomedullary difference in the FA values was more in group A. However, this difference was lower in group B and more so in group $\mathrm{C}$. This finding confirms the loss of corticomedullary differentiation in cases of allograft dysfunction.

On histopathology analysis, in group A patients $(n=23)$, biopsy showed normal finding or mild tubular atrophy and interstitial fibrosis in nearly all of them, except in two patients who showed moderate interstitial fibrosis and vacuolar tubulopathy due to calcineurin inhibitor toxicity. The FA of cortex and medulla in the patient with moderate interstitial fibrosis was lower than the mean FA values in this group ( 0.15 vs. 0.18 and 0.32 vs. 0.39 ). However, there was no significant difference found in the ADC values. However, the patient with calcineurin inhibitor toxicity showed no significant difference than the rest of the group A patients.

In group $B$ and $C(n=27)$, moderate interstitial fibrosis and tubular atrophy was seen in nine patients, severe interstitial fibrosis and tubular atrophy in six patients, vacuolar tubulopathy (calcineurin inhibitor toxicity) in four patients, chronic tubulointerstitial rejection in six patients, and acute $\mathrm{T}$ cell-mediated tubulointerstitial rejection in two patients.

\section{Discussion}

Kidney transplantation is the best form of long-term renal replacement therapy available for patients with end-stage

Table 1 Comparison between mean ADC and FA values of three groups

\begin{tabular}{|l|l|l|l|l|}
\hline Group & ADC cortex $\left(\times \mathbf{~ 1 0}^{-6} \mathrm{~mm}^{2} / \mathrm{s}\right)$ & ADC medulla $\left(\mathrm{x} 10^{-6} \mathrm{~mm}^{2} / \mathrm{s}\right)$ & FA cortex & FA medulla \\
\hline $\mathrm{A}(n=23)$ & $1,868 \pm 18$ & $1,814 \pm 24$ & $0.18 \pm 0.01$ & $0.39 \pm 0.02$ \\
\hline $\mathrm{B}(n=15)$ & $1,752 \pm 19$ & $1,694 \pm 21$ & $0.14 \pm 0.01$ & $0.26 \pm 0.02$ \\
\hline $\mathrm{C}(n=12)$ & $1,660 \pm 34$ & $1,612 \pm 33$ & $0.12 \pm 0.01$ & $0.23 \pm 0.02$ \\
\hline
\end{tabular}

Abbreviations: ADC, apparent diffusion coefficient; FA, fractional anisotropy.

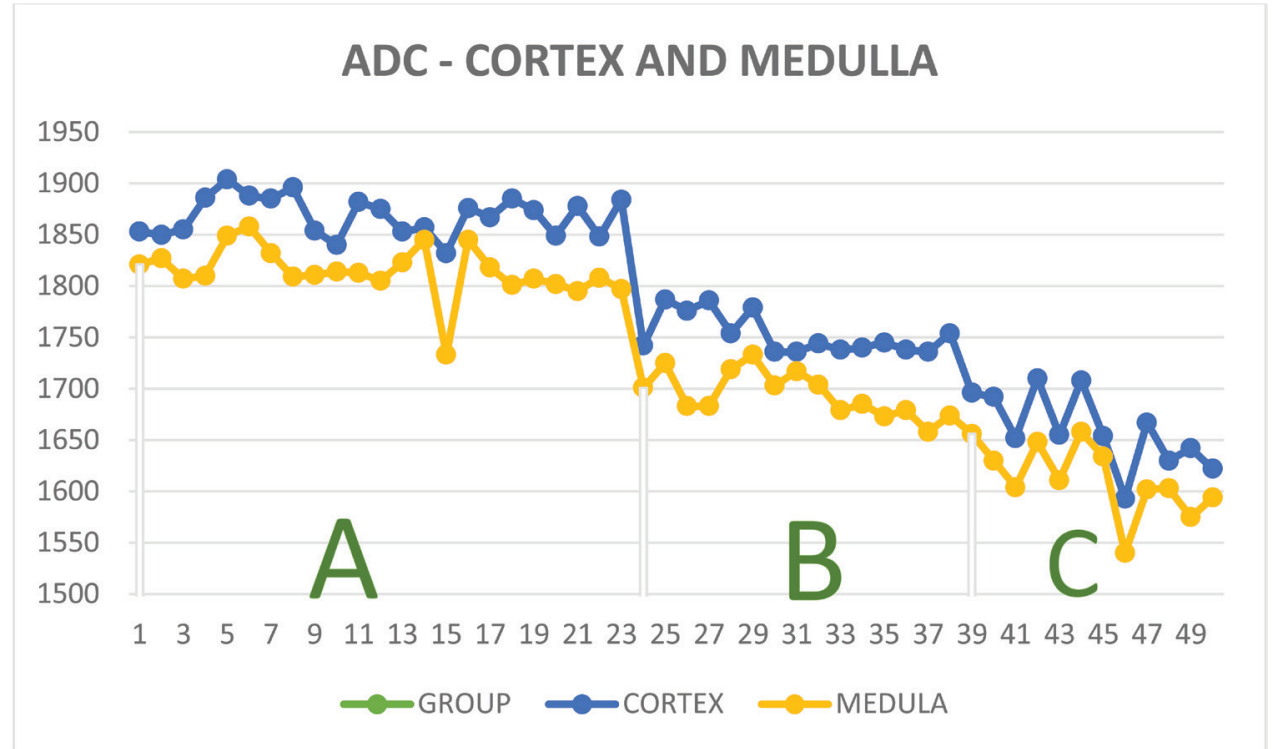

Fig. 3 Line chart diagram showing apparent diffusion coefficient (ADC) values and their difference between cortex and medulla in all three groups $(\mathbf{A}-\mathbf{C})$. 


\section{FA - CORTEX AND MEDULLA}

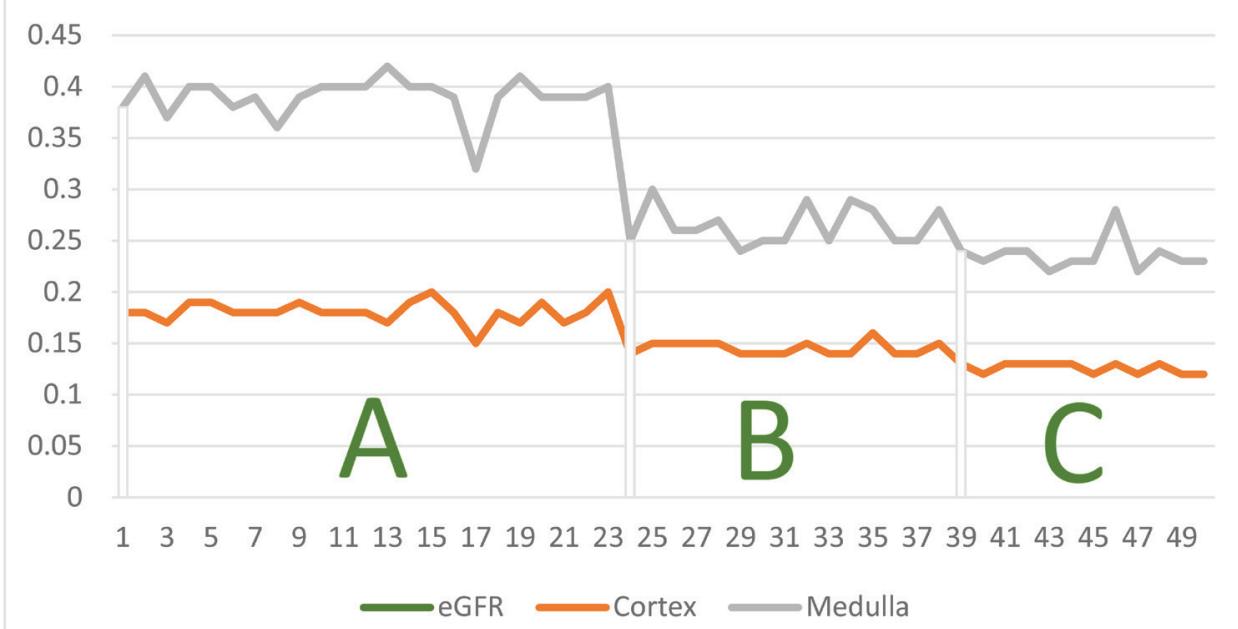

Fig. 4 Line chart diagram showing fractional anisotropy (FA) values and their difference between cortex and medulla in all three groups (A-C).

renal failure. Transplant dysfunction is one of the dreaded complications in these patients. Currently renal biopsy is the only standard diagnostic tool to evaluate allograft dysfunction, which is invasive and has its own complications.

Imaging plays a limited role in the functional evaluation of kidneys. Renal imaging is shifting from pure visualization of anatomy to assessment of physiologic and functional parameters of the kidney. ${ }^{7}$ New techniques in MRI such as BOLD and arterial spin labeling MRI, DWI, and DTI are emerging as promising noninvasive functional techniques in native as well as transplant kidney evaluation.

Initial studies in native kidneys have demonstrated high potential of diffusion imaging in the functional evaluation. However, the data are limited on the use of DWI and DTI in renal allograft evaluation. These results regarding the correlation between renal $A D C$ and kidney function are variable. Eisenberger et $\mathrm{al}^{8}$ demonstrated no correlation between ADC and eGFR, whereas other authors ${ }^{6,9}$ have reported a significant correlation between ADC and renal allograft function. An explanation for this variation is differences in imager geometry and use of various b values in the different studies. The choice of $b$ value affects ADC as found in many studies. ${ }^{10,11}$ Thoeny et $\mathrm{a}^{10}$ found no significant difference between the ADC values of the cortex and the medulla in healthy kidneys in low b value; but there was a significant difference in a high b value group. Low b values ( $<200$ seconds $\left./ \mathrm{mm}^{2}\right)$ contribute mainly to microperfusion. With high b values, the effect of perfusion is cancelled out, and the ADC value reflects pure diffusion. For this reason, the $\mathrm{b}$ value was selected to be 800 second $\mathrm{mm}^{2}$ in our study. As the medulla has more restricted diffusion due to radially oriented tubules and vessels, it showed low ADC values than the cortex. Zheng et a ${ }^{12}$ also concluded in their study that significant diffusion difference exists between the cortex and medulla in native kidneys. In their study, they found out that cortical diffusivity values were higher than medullary values and FA value was more in medulla than in cortex in native kidneys.
In our study, ADC values correlate well with eGFR value in renal allografts. We found a linear positive correlation between the eGFR and ADC of both cortex and medulla (-Figs. 5-7). Few other studies in transplant kidney ${ }^{13-15}$ also show that ADC values in patients with renal dysfunction were significantly lower than in patients with normal renal function and there was significant direct correlation between ADC and eGFR in these studies.

Kidney has anisotropic properties due to the presence of collecting ducts and tubules especially in the medulla. Anisotropic properties of tissues can be best evaluated using DTI, which, unlike DWI, allows the analysis of diffusion along multiple directions. The choice of b value influences the ADC, but not the FA. ${ }^{11}$ So it is clear that DTI is superior to DWI in kidneys and can detect early changes in tubular level. To our knowledge, there are only a few studies available regarding use of DTI in the evaluation of transplanted kidneys. ${ }^{16,17}$

In our study, we found a significant difference between cortical and medullary FA of transplant kidneys with good allograft function (group A-eGFR $>60 \mathrm{~mL} / \mathrm{min} / 1.73 \mathrm{~m}^{2}$ ) than those with poor function (group B and C, eGFR $<60$ $\left.\mathrm{mL} / \mathrm{min} / 1.73 \mathrm{~m}^{2}\right)$. FA values were significantly $(p<0.001)$ lower in the kidneys with poor allograft function. The corticomedullary difference in FA values was greater in group $A$, whereas it was significantly reduced in group B and C ( -Fig. 8). There are few theories to explain the lower FA values in poorly functioning allografts. ${ }^{18}$ One theory is "The cellular swelling and extracellular edema during tubulointerstitial rejection constrains the free water motions inside the tubules and diminishes the diffusion anisotropy resulting in low FA value." The other principle of FA reduction is "Due to the microcirculation influence on diffusion weighted images, decreased perfusion might have influence on the diffusion isotropy." These facts explain the low FA values in transplants with poor function.

In a similar study, Lanzman et al ${ }^{16}$ studied the correlation between the functional status of the transplant kidneys and 

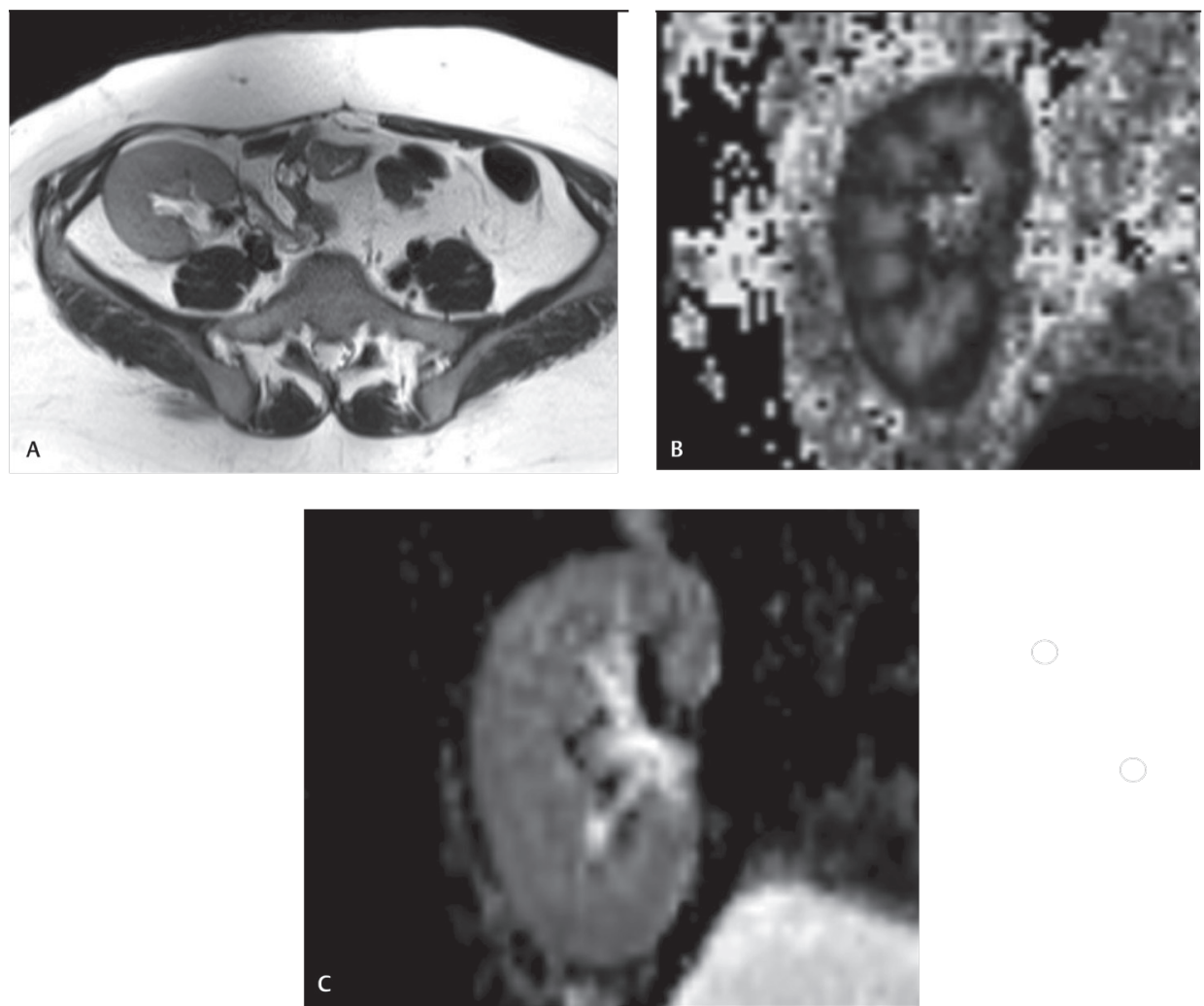

Fig. 5 MR images in a 50-year-old female patient (from group A) 1 year after renal transplantation. (A) T2 half-Fourier acquisition single-shot turbo spin echo image shows the normal sized transplant kidney in the right iliac fossa. (B) Diffusion tensor imaging fractional anisotropy (FA) maps of the graft show good corticomedullary differentiation (mean cortical FA $=0.18$ and medullary $F A=0.39$ ). (C) Apparent diffusion coefficient (ADC) map shows high signal intensity (mean cortical $A D C=1,848 \times 10^{-6} \mathrm{~mm}^{2} / \mathrm{s}$ and medullary $A D C=1,808 \times 10^{-6} \mathrm{~mm}^{2} / \mathrm{s}$ ).
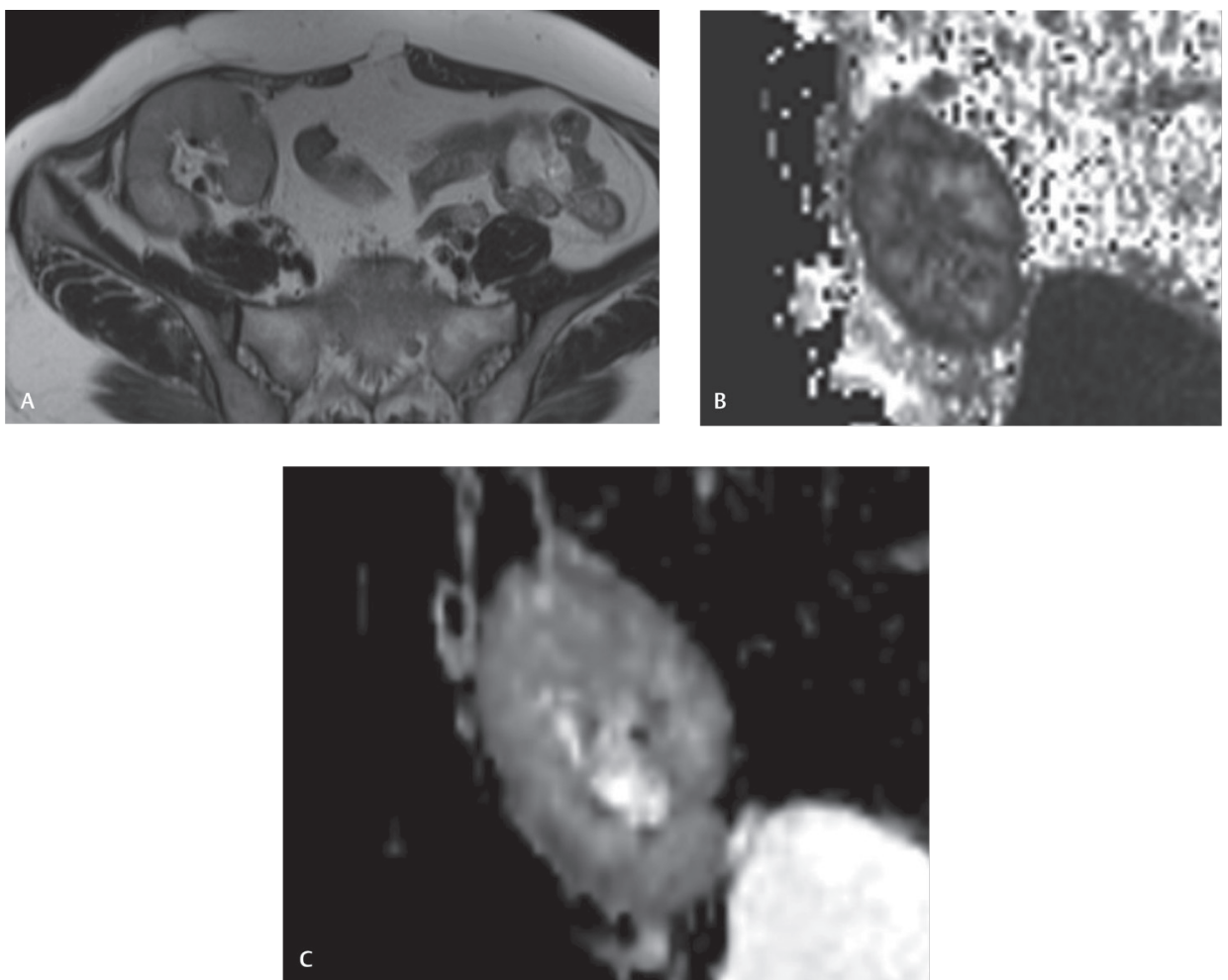

Fig. 6 Images in a 61-year-old female patient (from group B) 5 years after renal transplantation. (A) T2 half-Fourier acquisition single-shot turbo spin echo image shows transplant kidney in the right iliac fossa. (B) Diffusion tensor imaging fractional anisotropy (FA) maps of the graft show reduction in corticomedullary differentiation (mean cortical FA $=0.14$ and medullary FA $=0.29$ ). (C) Apparent diffusion coefficient (ADC) map shows homogenous low intensity of the graft (mean cortical $A D C=1,740 \times 10^{-6} \mathrm{~mm}^{2} / \mathrm{s}$ and medullary $A D C=1,685 \times 10^{-6} \mathrm{~mm}^{2} / \mathrm{s}$ ). 

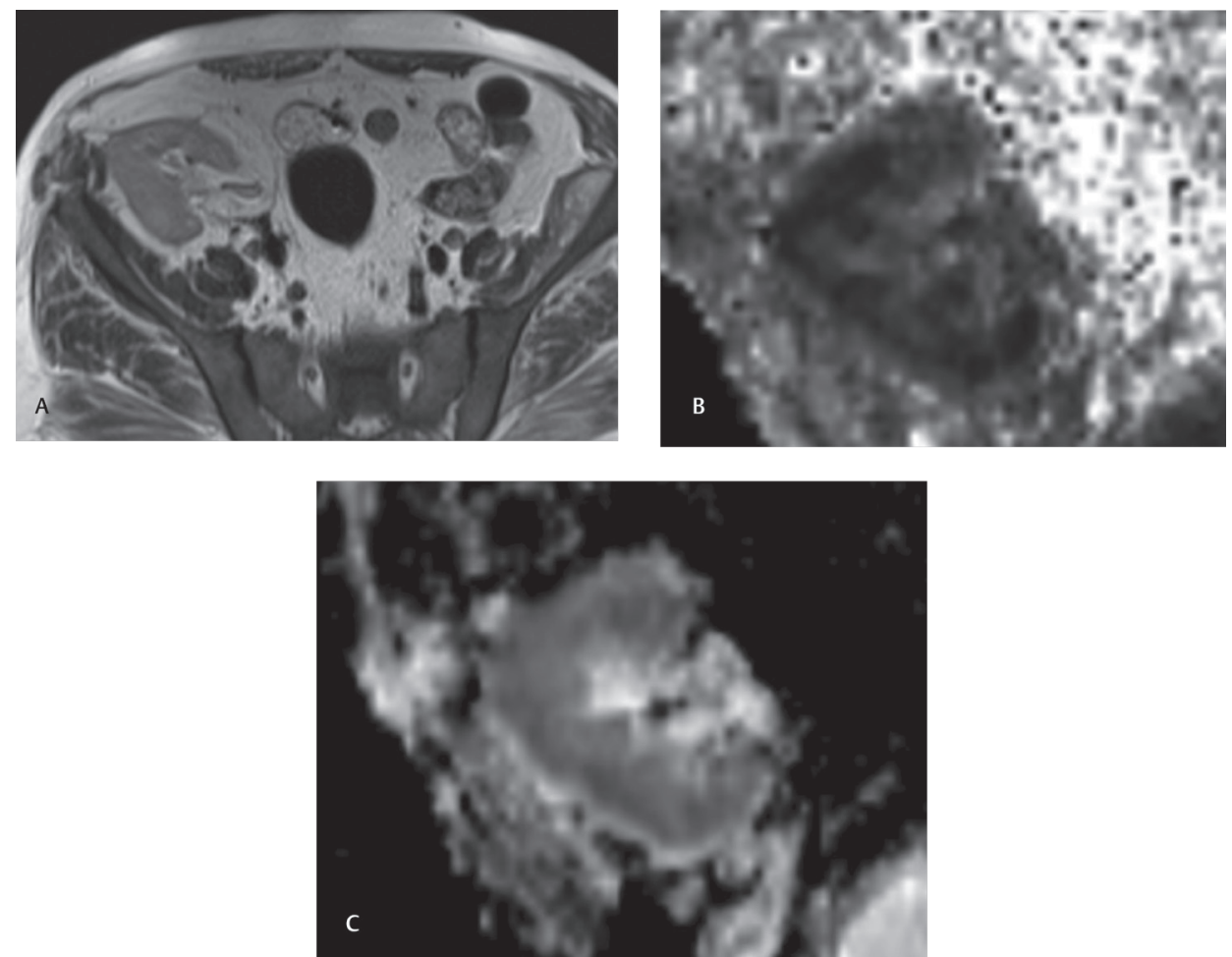

Fig. 7 Images in a 60-year-old male patient (from group C) 10 years after renal transplantation. (A) T2 half-Fourier acquisition single-shot turbo spin echo image shows transplant kidney in the right iliac fossa. (B) Diffusion tensor imaging fractional anisotropy (FA) maps of the graft show poor corticomedullary differentiation with low FA values (mean cortical FA $=0.12$ and medullary FA $=0.23$ ). (C) Apparent diffusion coefficient $(A D C)$ map also shows low values (mean cortical $A D C=1,622 \times 10^{-6} \mathrm{~mm}^{2} / \mathrm{s}$ and medullary $A D C=1,594 \times 10^{-6} \mathrm{~mm}^{2} / \mathrm{s}$ ).
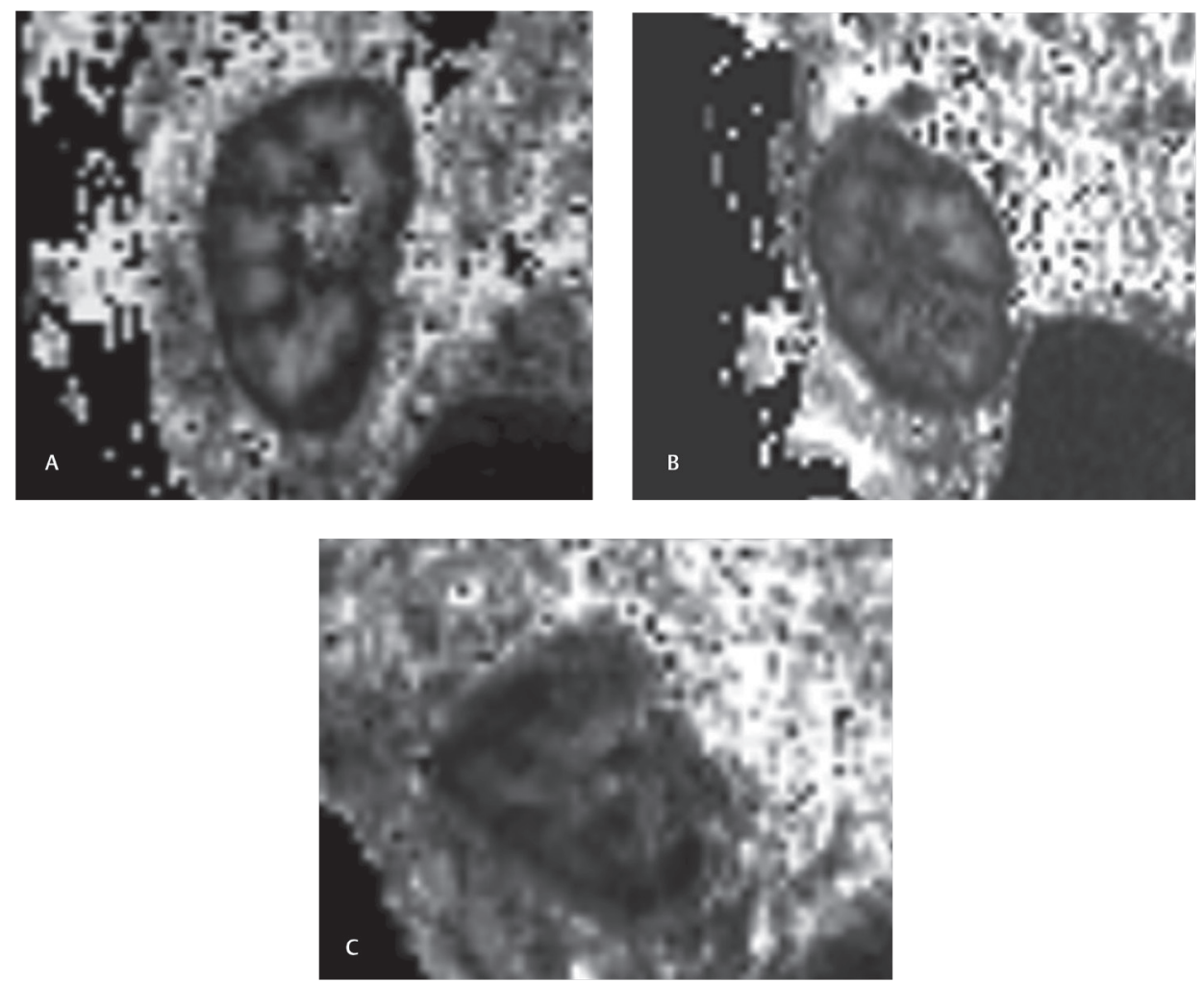

Fig. 8 Comparison between the fractional anisotropy maps of different patients from group A, B, and C (A, B, and C, respectively). Note the poor corticomedullary differentiation in patients from group B and C. 
DTI values (ADC and FA) in 40 patients. They divided the patients into two groups, those with eGFR above 30 (group A) and below $30 \mathrm{~mL} / \mathrm{min} / 1.73 \mathrm{~m}^{2}$ (group B). In that study, they found mean FA of the renal medulla and cortex was significantly higher in group A compared with group B. However, in our study it's clear that even the patients with mild to moderately decreased function (eGFR 30-60 mL/min/1.73 $\mathrm{m}^{2}$, group $B$ in our study) had significantly decreased ADC and FA values compared with patients with eGFR $>60 \mathrm{~mL} / \mathrm{min} / 1.73 \mathrm{~m}^{2}$.

In our study, we found a strong positive linear correlation between the ADC values of medulla and eGFR $(r=0.91)$. Similarly, ADC and FA of the cortex $(r=0.87)$ and $(r=0.80)$ also showed strong positive correlation with eGFR in group $B$ and $C$. This means that reduction in the eGFR causes low DTI values. However, FA values in medulla showed moderate positive correlation with eGFR $(r=0.65)$.

The ADC and FA values of patients in group $B$ were significantly lower than patients in group A $(p<0.001)$. Similarly, there was a significant difference in DTI values between group B and C.

Histopathological findings in the group of the dysfunctional allografts were inhomogeneous. Thus, the potential of DTI to differentiate between pathologic conditions (such as interstitial fibrosis, tubulointerstitial rejection, or calcineurin inhibitor toxicity) cannot be derived from our results.

We had few limitations in our study, such as the correlations between the post-transplantation time, age of the kidney donor, and type of donor (living or cadaveric), that were not assessed. The ROIs that were placed to calculate ADC and FA covered only part and not entire of the transplant kidney.

\section{Conclusion}

We have studied the use of DTI for the functional evaluation of transplant kidneys, measured by eGFR. ADC and FA values in the renal cortex and medulla exhibit a good correlation with allograft function. The mean ADC and FA of the transplants show good positive correlation with eGFR in patients with graft dysfunction (group B and C) and were significantly lower than those with good function. FA values seem to be more sensitive than eGFR and ADC for the early pathological changes in the graft. The results of our study correlating the DTI parameters and eGFR using 3-Tesla MRI suggest that DTI could be a reliable noninvasive tool to evaluate the allograft functional status after renal transplantation for early detection of microstructural derangements and indicate the time to perform biopsy. In view of high reproducibility, lack of need for contrast agent administration, and the high sensitivity to microstructural changes, it is also an excellent tool for treatment follow-up. However, large-scale studies linked with histopathologic analysis are needed to prove the above results.

\section{Conflict of Interest}

None declared.

\section{References}

1 Abecassis M, Bartlett ST, Collins AJ, et al. Kidney transplantation as primary therapy for end-stage renal disease: a National Kidney Foundation/Kidney Disease Outcomes Quality Initiative (NKF/KDOQITM) conference. Clin J Am Soc Nephrol 2008;3(2):471-480

2 Agarwal SK, Srivastava RK. Chronic kidney disease in India: challenges and solutions. Nephron Clin Pract 2009;111(3):c197-c203, discussion c203

3 Sund S, Reisaeter AV, Fauchald P, Bentdal O, Hall KS, Hovig T. Living donor kidney transplants: a biopsy study 1 year after transplantation, compared with baseline changes and correlation to kidney function at 1 and 3 years. Nephrol Dial Transplant 1999;14(10):2445-2454

4 Michaely HJ, Sourbron S, Dietrich O, Attenberger U, Reiser MF, Schoenberg SO. Functional renal MR imaging: an overview. Abdom Imaging 2007;32(6):758-771

5 Laissy JP, Idée JM, Fernandez P, Floquet M, Vrtovsnik F, Schouman-Claeys E. Magnetic resonance imaging in acute and chronic kidney diseases: present status. Nephron Clin Pract 2006;103(2):c50-c57

6 Thoeny HC, Zumstein D, Simon-Zoula S, et al. Functional evaluation of transplanted kidneys with diffusion-weighted and BOLD MR imaging: initial experience. Radiology 2006;241(3):812-821

7 Chandarana H, Lee VS. Renal functional MRI: are we ready for clinical application? AJR Am J Roentgenol 2009;192(6):1550-1557

8 Eisenberger U, Thoeny HC, Binser T, et al. Evaluation of renal allograft function early after transplantation with diffusion-weighted MR imaging. Eur Radiol 2010;20(6):1374-1383

9 Blondin D, Lanzman RS, Klasen J, et al. Diffusion-attenuated MRI signal of renal allografts: comparison of two different statistical models. AJR Am J Roentgenol 2011;196(6):W701-5

10 Thoeny HC, De Keyzer F, Oyen RH, Peeters RR. Diffusionweighted MR imaging of kidneys in healthy volunteers and patients with parenchymal diseases: initial experience. Radiology 2005;235(3):911-917

11 Notohamiprodjo M, Dietrich O, Horger W, et al. Diffusion tensor imaging (DTI) of the kidney at 3 Tesla-feasibility, protocol evaluation and comparison to 1.5 Tesla. Invest Radiol 2010;45(5):245-254

12 Zheng Z, Shi H, Zhang J, Zhang Y. Renal water molecular diffusion characteristics in healthy native kidneys: assessment with diffusion tensor MR imaging. PLoS One 2014;9(12):e113469

13 Blondin D, Lanzman RS, Mathys C, et al. [Functional MRI of transplanted kidneys using diffusion-weighted imaging]. RoFo FortschrGebRontgenstrNuklearmed2009;181(12):1162-1167

14 Abou-El-Ghar ME, El-Diasty TA, El-Assmy AM, Refaie HF, Refaie AF, Ghoneim MA. Role of diffusion-weighted MRI in diagnosis of acute renal allograft dysfunction: a prospective preliminary study. Br J Radiol 2012;85(1014):e206-e211

15 Kaul A, Sharma RK, Gupta RK, et al; Jaisuresh. Assessment of allograft function using diffusion-weighted magnetic resonance imaging in kidney transplant patients. Saudi J Kidney Dis Transpl 2014;25(6):1143-1147

16 Lanzman RS, Ljimani A, Pentang G, et al. Kidney transplant: functional assessment with diffusion-tensor MR imaging at 3T. Radiology 2013;266(1):218-225

17 Hueper K, Gutberlet M, Rodt T, et al. Diffusion tensor imaging and tractography for assessment of renal allograft dysfunction-initial results. Eur Radiol 2011;21(11):2427-2433

18 Fukuda Y, Ohashi I, Hanafusa K, et al. Anisotropic diffusion in kidney: apparent diffusion coefficient measurements for clinical use. J Magn Reson Imaging 2000;11(2):156-160 